\title{
Time-Dependent Numerical Modelling of Tides in the Middle Atmosphere
}

\author{
Takehiko Aso \\ Dept. Electrical Eng., Kyoto University, Kyoto 606, Japan
}

(Received July 7, 1992; Revised November 12, 1992)

\begin{abstract}
Atmospheric solar tides observed in the middle atmosphere reveal various temporal and spatial variability, and snap-shot observations sometimes deviate from "average" tidal features. To evaluate transient behavior of this global-scale wave, time evolution of solar atmospheric tide has been investigated by means of time-dependent numerical modelling. A spectral method is applied to the primitive equation system, in which horizontal momentum equations, thermodynamic equation and lower boundary condition for the geopotential height are solved by the appropriate time integration scheme, with the continuity and hydrostatic equations solved downward and upward, respectively, in the altitude direction. Boundary conditions assume non-slip hypothesis below and diffusion-dominated spongy layer above.

Time evolution of $(2,2)$ mode for the forcing with rise time of 2 days exhibits set-up time of 20 days or more, and vertical group propagation in the lower altitude region is shown to be comparable to theoretical value of about $0.6 \mathrm{~m} / \mathrm{sec}$. For $(1,-2)$ mode, set-up time is short, and oscillation of complex amplitude with period of about 1.2 day is detected which might be due to beats with excited $5 \sim 7$-day period normal mode wave. Propagating $(1,1)$ mode shows smaller vertical group velocity for the rise-up of tidal structure. Semidiurnal tide excited non-linearly by the diurnal thermotidal forcing shows horizontal velocity component of some $10 \mathrm{~m} / \mathrm{sec}$ at around $90 \mathrm{~km}$ which might affect the linear description of semidiurnal tide. These and other findings are informative to delineate tidal variability quantitatively.
\end{abstract}

\section{Introduction}

Atmospheric tide reveals itself in the neutral motion field and temperature perturbations in the middle to upper atmosphere. The tidal wave is predominantly excited by the insolation absorption of water vapor and ozone in the lower atmosphere and gains its amplitude in proportion to the inverse square root of background atmospheric density and becomes a significant wave component in the observations by radar and optical probing in the middle atmosphere. Tidal components observed in this region sometimes indicate inherent variability from day-to-day to seasonal or yearly time scales. These variabilities are attributable to the change in forcing, background atmospheric parameters as temperature and mean zonal wind, dissipation due to tidal and gravity wave breaking, superposition of nonmigrating local tide, nonlinear interaction with tide and other wave modes in the course of excitation and propagation into the upper atmosphere. Among others, the effect of mean wind or background atmospheres on tides has been clarified by LINDZEN and Hong (1974), Aso et al. (1981, 1987), ViAL (1986), ForBes $(1982 \mathrm{a}, \mathrm{b})$ and others in terms of steady state modelling, and long-term tidal climatology for the semidiurnal tide has recently been summarized by FORBES and VIAL (1989).

Tidal propagation in the middle atmosphere is envisaged as a system response of rotating, shallow atmosphere to external solar (or lunar) forcing, and it is worthwhile to estimate transient behaviors of tides so as to resolve its day-to-day or short-term variability. MrYaHARA (1981) first calculated linear evolution of diurnal tide in connection with mean wind acceleration by momentum transport of dissipating tide. VIAL et al. (1991) recently calculated some transient aspects of tides excited by respective tidal modes and identified rise time of tidal structure based 
on their vertical propagation. They solved equations for the poloidal and toroidal vectors which describe momentum field. Also, Wu et al. (1989) showed the effect of nonlinear interactions of diurnal tide with mean wind which is suggestive of possible discrepancies of tidal feature from simple theoretical prediction obtained so far by the linearized steady tidal studies.

In this paper, mechanistic modelling of the time evolution of solar atmospheric tide has been given using the time-dependent spectral model based on the primitive equation system. These calculations for the linearized regime indicate rise-up of dominant diurnal $(1,-2),(1,1)$ and semidiurnal $(2,2)$, and $(2,4)$ components to imposed forcing with rise-time of 2 days. These results show vertical group propagation as in VIAL et al. (1991). Oscillations of complex amplitude are evidently detected as a transient response which might account for short-term variability due to change in forcing and propagation condition, and also superposition of normal mode waves. Then, excitation of semidiurnal components by diurnal $(1,1)$ and $(1,-2)$ modes is estimated using the nonlinear regime of the present model. The amplitude of nonlinearly excited semidiurnal component at steady state is comparable to those of linearly forced semidiurnal component at higher altitudes. This might complicate the interpretation of observed semidiurnal component by a simple theoretical prediction.

\section{Model Descriptions}

\subsection{Basic equations}

A set of primitive equations for the present mechanistic modelling is the horizontal momentum equations, the first law of thermodynamics, the hydrostatic approximation and the continuity equation (Holton, 1975),

$$
\begin{gathered}
\frac{\partial u}{\partial t}+\frac{1}{a \sin \theta} \frac{\partial}{\partial \theta}\left(u^{2} \sin \theta\right)+\frac{1}{a \sin \theta} \frac{\partial}{\partial \phi}(u v)-\frac{1}{a \tan \theta} v^{2} \\
+\frac{1}{p} \frac{\partial}{\partial z^{*}}\left(p w^{*} u\right)-2 \omega v \cos \theta+g \frac{\partial z}{a \partial \theta} \\
-\frac{H_{s}^{2}}{p} \frac{\partial}{\partial z^{*}}\left(\frac{p}{H^{2}} \nu_{e d d y} \frac{\partial u}{\partial z^{*}}\right)-\frac{g H_{s}}{p} \frac{\partial}{\partial z^{*}}\left(\mu_{m o l} \frac{H_{s}}{H} \frac{\partial u}{\partial z^{*}}\right)=0 \\
\frac{\partial v}{\partial t}+\frac{1}{a \sin \theta} \frac{\partial}{\partial \theta}(u v \sin \theta)+\frac{1}{a \sin \theta} \frac{\partial v^{2}}{\partial \phi}+\frac{1}{a \tan \theta} u v \\
+\frac{1}{p} \frac{\partial}{\partial z^{*}}\left(p w^{*} v\right)+2 \omega u \cos \theta+2 \omega w^{*} \sin \theta+\frac{g}{a \sin \theta} \frac{\partial z}{\partial \phi} \\
-\frac{H_{s}^{2}}{p} \frac{\partial}{\partial z^{*}}\left(\frac{p}{H^{2}} \nu_{e d d y} \frac{\partial v}{\partial z^{*}}\right)-\frac{g H_{s}}{p} \frac{\partial}{\partial z^{*}}\left(\mu_{m o l} \frac{H_{s}}{H} \frac{\partial v}{\partial z^{*}}\right)=0 \\
\frac{\partial T}{\partial t}+u \frac{\partial T}{a \partial \theta}+\frac{v}{a \sin \theta} \frac{\partial T}{\partial \phi}+w^{*}\left(\frac{\partial T}{\partial z^{*}}+\frac{R T}{C_{p} H_{s}}\right)-\frac{Q}{C_{p}}+\alpha_{i} T \\
-\frac{g H_{s}^{2}}{C_{p} p} \frac{\partial}{\partial z^{*}}\left(\frac{g}{R T} K \frac{\partial T}{\partial z^{*}}\right)-\frac{H_{s}^{2}}{p} \frac{\partial}{\partial z^{*}}\left\{\frac{p K_{e d d y}}{H^{2}}\left(\frac{\partial T}{\partial z^{*}}+\frac{R T}{C_{p} H_{s}}\right)\right\}=0 \\
\frac{1}{a \sin \theta} \frac{\partial v}{\partial \phi}+\frac{R}{a \sin \theta} \frac{\partial}{\partial \theta}(u \sin \theta)+\frac{\partial w^{*}}{\partial z^{*}}-\frac{w^{*}}{H_{s}}=0 \\
T
\end{gathered}
$$


The log-pressure co-ordinate system is used, and the symbols are $t$ : time, $\theta$ : colatitude, $\phi$ : east longitude, $z^{*}$ : a measure of height $H_{s} \ln \left(p_{s} / p\right)$, $u$ : northerly wind velocity, $v$ : westerly wind velocity, $w^{*}$ : a measure of upward vertical velocity $d z^{*} / d t, p$ : pressure, $\rho$ : atmospheric density, $T$ : temperature, $z$ : geopotential height, $p_{s}$ : constant reference pressure, $H_{s}$ : constant scale height, $\omega$ : earth rotation rate, $g$ : acceleration of gravity, $a$ : earth radius, $H$ : scale height $R T / g, \nu_{\text {eddy }}$ : eddy diffusion coefficient, $\mu_{m o l}$ : dynamic molecular viscosity coefficient, $K_{\text {eddy }}$ : eddy thermal conductivity, $K$ : molecular thermal conductivity coefficient, $R$ : gas constant for dry air, $C_{p}$ : heat capacity at constant pressure, $\alpha_{i}$ : Newtonian cooling rate, $Q$ : Solar thermal heating per unit mass. The temperature $T$ is divided into basic static state $T_{0}\left(z^{*}\right)$ and departure $T_{1}$, and correspondingly geopotential height $z$ is expressed as the sum of basic state $z_{0}\left(z^{*}\right)$ and departure $z_{1} . T_{0}$ and $z_{0}$ are implicitly assumed to be maintained by the zonal mean solar heating $Q_{0}$.

Non-dimensionalization of each variable as

$$
\begin{array}{lll}
x=\frac{z^{*}}{H_{s}}, & y=\cos \theta, & \left(\begin{array}{c}
u \\
v
\end{array}\right)=\frac{g}{2 \omega \sin \theta}\left(\begin{array}{c}
\hat{u} \\
\hat{v}
\end{array}\right), \\
\hat{t}=2 \omega t, & w^{*}=\frac{g H_{s}}{2 \omega a} \hat{w}, & T=\frac{a g}{R} \hat{T}, \\
z=a \hat{z}, & H=\frac{R T}{g}, & \hat{\nu}=\frac{1}{2 \omega H^{2}}\left(\nu_{e d d y}+\frac{\mu_{m o l}}{\rho}\right), \\
K_{\text {eddy }}=2 \omega H^{2} \hat{K}_{e}, & \frac{K}{\rho}=2 \omega H^{2} C_{p} \hat{K}, & Q=2 \omega a g \frac{C_{p}}{R} \hat{Q} \\
\hat{\alpha}_{i}=\frac{\alpha_{i}}{2 \omega}, &
\end{array}
$$

and scaling of velocity field and departures of temperature and height as

$$
\left(\begin{array}{c}
\hat{u} \\
\hat{v} \\
\hat{w} \\
\hat{z}_{1} \\
\hat{T}_{1}
\end{array}\right)=\left(\begin{array}{c}
\tilde{u} \\
\tilde{v} \\
\tilde{w} \\
\tilde{z} \\
\tilde{T}
\end{array}\right) e^{x / 2}
$$

give the following set of equations.

$$
\begin{aligned}
\frac{\partial \tilde{u}}{\partial \hat{t}}= & {\left[\frac{C y}{1-y^{2}} \tilde{u}^{2} e^{x / 2}+C \frac{\partial \tilde{u}^{2}}{\partial y} e^{x / 2}-\frac{C}{1-y^{2}} \frac{\partial(\tilde{u} \tilde{v})}{\partial \phi} e^{x / 2}-C \frac{\partial(\tilde{w} \tilde{u})}{\partial x} e^{x / 2}\right.} \\
& \left.+\frac{C y}{1-y^{2}} \tilde{v}^{2} e^{x / 2}\right]+y \tilde{v}+\left(1-y^{2}\right) \frac{\partial \tilde{z}}{\partial y}+\frac{1}{p} \frac{\partial}{\partial x}\left(p \hat{\nu} \frac{\partial \hat{u}}{\partial x}\right) e^{-x / 2} \\
\frac{\partial \tilde{v}}{\partial \hat{t}}= & {\left[C \frac{\partial(\tilde{u} \tilde{v})}{\partial y} e^{x / 2}-\frac{C}{1-y^{2}} \frac{\partial \tilde{v}^{2}}{\partial \phi} e^{x / 2}-C \frac{\partial(\tilde{w} \tilde{v})}{\partial x} e^{x / 2}\right] } \\
& -y \tilde{u}-\delta\left(1-y^{2}\right) \tilde{w}-\frac{\partial \tilde{z}}{\partial \phi}+\frac{1}{p} \frac{\partial}{\partial x}\left(p \hat{\nu} \frac{\partial \hat{v}}{\partial x}\right) e^{-x / 2}, \\
\frac{\partial \tilde{T}}{\partial \hat{t}=} & {\left[C \tilde{u} \frac{\partial \tilde{T}}{\partial y} e^{x / 2}-C \frac{\tilde{v}}{1-y^{2}} \frac{\partial \tilde{T}}{\partial \phi} e^{x / 2}-C \tilde{w}\left(\frac{\partial \tilde{T}}{\partial x}+\frac{\tilde{T}}{2}+\frac{R}{C_{p}} \tilde{T}\right) e^{x / 2}\right] } \\
& -C\left(\frac{d \hat{T}_{0}}{d x}+\frac{R}{C_{p}} \hat{T}_{0}\right) \tilde{w}+\hat{Q} e^{-x / 2}+\frac{1}{p} \frac{\partial}{\partial x}\left(p \hat{K} \frac{\partial \hat{T}}{\partial x}\right) e^{-x / 2}
\end{aligned}
$$




$$
\begin{aligned}
&-\hat{\alpha}_{i} \tilde{T}+\frac{1}{p} \frac{\partial}{\partial x}\left\{p \hat{K}_{e}\left(\frac{\partial \hat{T}}{\partial x}+\frac{R}{C_{p}} \hat{T}\right)\right\} e^{-x / 2} \\
& \frac{\partial \tilde{z}}{\partial x}+\frac{\tilde{z}}{2}=\tilde{T} \\
&-\frac{\partial \tilde{u}}{\partial y}+\frac{1}{1-y^{2}} \frac{\partial \tilde{v}}{\partial \phi}+\frac{\partial \tilde{w}}{\partial x}-\frac{\tilde{w}}{2}=0
\end{aligned}
$$

where $C=g / 4 \omega^{2} a, \delta=H_{s} / a$.

\subsection{Spectral model}

Dependent variables $\tilde{u}, \tilde{v}, \tilde{w}, \tilde{z}, \tilde{T}$ are expressed in terms of spherical harmonics as

$$
\psi(x, y, \phi, t)=\sum_{s} \sum_{l \geq|s|} \Psi_{l, s}(x, t) P_{l, s}(y) e^{j s \phi}
$$

where $P_{l, s}$ is the associated Legendre function of normalized Schmidt-type of order $s$, degree $l$, and $s$ is the zonal wavenumber. Equation (13) is substituted in Eqs. (8) through (12) and coupled partial differential equations for each component $\Psi_{l, s}(x, t)$ are solved numerically for the assumed $(l, s)$ component of forcing.

The $(l, s)$ component of linear terms is analytically formulated. For example, a term (1 $\left.y^{2}\right) \partial \tilde{z} / \partial y$ in Eq. (8) is expressed as (HALtiner and Williams, 1980)

$$
\int\left(1-y^{2}\right) \frac{\partial}{\partial y}\left[\sum_{l^{\prime}} Z_{l^{\prime}, s} P_{l^{\prime}, s}\right] P_{l, s} d y=-(l-1) \gamma_{l, s} Z_{l-1, s}+(l+2) \gamma_{l+1, s} Z_{l+1, s}
$$

where $\gamma_{l, s} \equiv \sqrt{\frac{l^{2}-s^{2}}{4 l^{2}-1}}$.

Spectral components of nonlinear terms are obtained by the transform model, in which nonlinear quantities are first calculated in terms of reconstructed values in the physical space and Fourier-Legendre decomposed. This is more efficient than the interaction coefficient model in case a large number of expansion coefficients are included in the modelling. Also, in calculating $(l, s)$ spectral components as $\partial(\tilde{u} \tilde{v}) / \partial y$, tables of $\int d P_{l, s} / d y \cdot P_{l^{\prime}, s} d y$ which has been prepared by the REDUCE algebraic calculation and numerical integration are used. Latitudinal integration was carried out by the Gaussian quadrature formulas for 32 latitudinal points.

\subsection{Boundary condition}

Constraints at the upper boundary $z^{*}=z_{\text {top }}^{*}$ impose zero vertical gradient of dependent variables in the diffusion dominated spongy layer. Thus,

$$
\frac{\partial u}{\partial z^{*}}=\frac{\partial v}{\partial z^{*}}=\frac{\partial T_{1}}{\partial z^{*}}=\frac{\partial w}{\partial z^{*}}=0
$$

If $\psi$ stands for either of $\tilde{u}, \tilde{v}, \tilde{T}, \tilde{w}$, this leads to

$$
\frac{\partial \psi}{\partial x}+\frac{1}{2} \psi=0
$$

For the horizontal wind velocity and temperature perturbations near the ground $z^{*}=0$, we assume non-slip boundary condition,

$$
u=v=T_{1}=0 .
$$


(a)

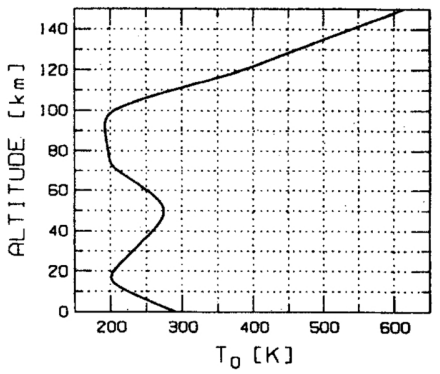

(b)

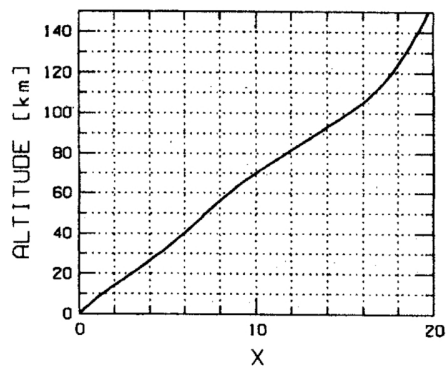

Fig. 1. (a) Basic temperature profile $T_{0}$ and (b) the relation between $z$ and $x=\ln \frac{p_{s}}{p}=\int_{0}^{z} \frac{d z}{H}$ based on the basic temperature profile $T_{0}$.

Vertical velocity of air parcel approaches zero at $z^{*}=0$, viz., $\frac{d z_{1}}{d t}=0$ gives

$$
\frac{\partial z_{1}}{\partial t}+\frac{u}{a} \frac{\partial z_{1}}{\partial \theta}+\frac{v}{a \sin \theta} \frac{\partial z_{1}}{\partial \phi}+w^{*} \frac{\partial z_{1}}{\partial z^{*}}=0
$$

With the use of hydrostatic equation, and non-dimensionalization as above, along with the nonslip condition for $u$ and $v$, this reduces to

$$
\frac{\partial \tilde{z}}{\partial \hat{t}}=-C \tilde{w}\left(\frac{d \hat{z}_{0}}{d x}+\frac{\partial \hat{z}_{1}}{\partial x}\right)
$$

\subsection{Atmospheric model}

The altitude profile of basic state atmospheric temperature is shown in Fig. 1(a) which is similar to the one used in the previous steady-state tidal modelling (Aso et al., 1981, 1987). Solar thermotidal forcing is superposed to the zonal mean solar heating which maintains the basic temperature structure. The relation between $z$ and $x=\ln \left(p_{s} / p\right)=\int_{0}^{z} d z / H$ based on the adopted temperature profile is shown in Fig. 1(b). The uppermost height is $x=19.5$ at $z=150$ $\mathrm{km}$.

Dissipations are introduced by the transport of heat and momentum by molecular and eddy diffusion and Newtonian cooling for the infrared radiation. Molecular and eddy viscosity coefficients $\mu_{m o l} / \rho$ and $\nu_{e d d y}$, and also Newtonian cooling coefficient $\alpha_{i}$ are respectively shown in Fig. 2(a)-(c). Molecular and eddy conductivity coefficients $K / \rho$ and $K_{\text {eddy }}$ are given as $1.36 \times 10^{3} \mu_{m o l} / \rho$ and $1.36 \nu_{e d d y}$, respectively. These dissipation profiles are adapted from the model given by FORBES (1982a) and used in the above-mentioned steady-state calculation.

\subsection{Numerical scheme}

Two time marching schemes are used in the present calculation, the Matsuno or Euler backward scheme (Matsuno, 1966) and the leapfrog scheme. The Matsuno method is stable, avoiding stable but sophisticated implicit scheme (HALTINER and WILliams, 1980), but requires twice the computing time relative to the leapfrog method. For the differential equation of the form $d \psi / d t=F(\psi(t), t)$, the algorithm calculates $\psi(t+\Delta t)$ from the value $\psi(t)$ in two steps as

$$
\left.\begin{array}{l}
\psi^{*}(t+\Delta t)=\psi(t)+F(\psi(t), t) \Delta t \\
\psi(t+\Delta t)=\psi(t)+F\left(\psi^{*}(t+\Delta t), t+\Delta t\right) \Delta t
\end{array}\right\}
$$


(a)

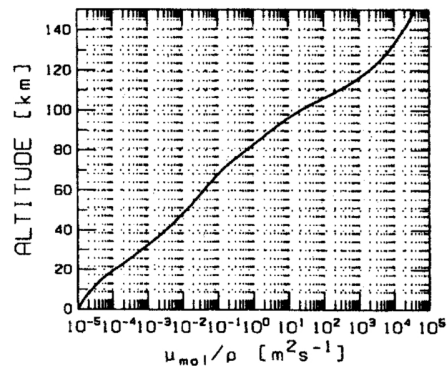

(c)

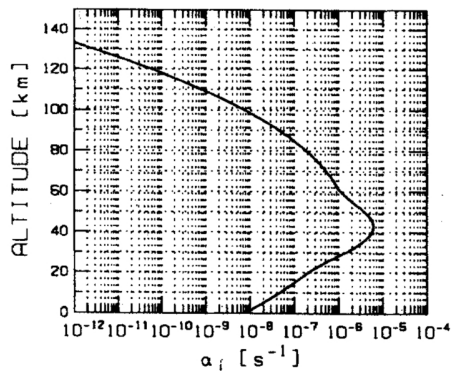

(b)

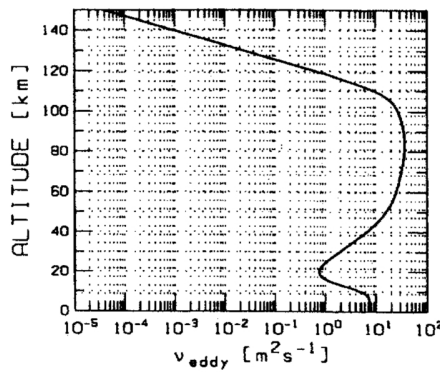

(d)
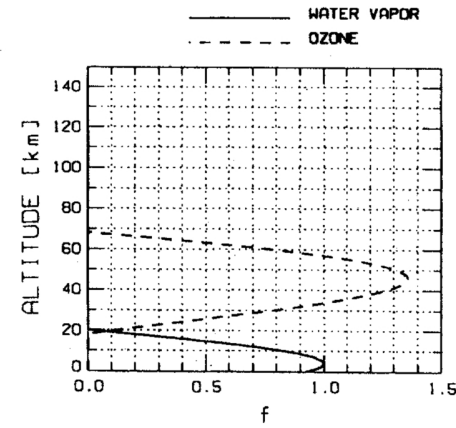

Fig. 2. Altitude profiles of (a) molecular and (b) eddy viscosity coefficients $\frac{\mu_{m o l}}{\rho}$ and $\nu_{e d d y}$, (c) Newtonian cooling coefficient $\alpha_{i}$ and (d) ozone and water vapor thermal forcing.

If we consider the oscillation equation $d \psi / d t=i \omega \psi$ and assume the solution at $n \Delta t$ to be $\psi_{n}=c \lambda^{n \Delta t}$, amplification factor is

$$
\lambda^{\Delta t}=1+i \omega \Delta t-\omega^{2}(\Delta t)^{2}
$$

and is stable, i.e., $\left|\lambda^{\Delta t}\right|<1$ so long as $\omega \Delta t \leq 1$. This also indicates inherent dissipating effect in the algorithm. For the diurnal oscillation, $\omega=7.292 \times 10^{-5} \mathrm{sec}^{-1}$ and e-folding time $T_{1 / e}$ for the time step $\Delta t=2.5$ and $5 \mathrm{~min}$ is 29 and 15 days, respectively. These values are fairly large, suggesting no appreciable attenuation for this wave component. While for the semidiurnal oscillation,

$$
T_{1 / e}=\frac{-1}{\ln \left|\lambda^{\Delta t}\right|} \simeq\left\{\begin{array}{lll}
4.2 \times 10^{3} \text { (steps) } \simeq 7.3(\text { day }) & (\Delta t=2.5 \mathrm{~min}) \\
1.0 \times 10^{3} \text { (steps) } \simeq 3.6(\text { day }) & (\Delta t=5 \mathrm{~min}) \\
2.6 \times 10^{2} \text { (steps) } \simeq 1.8(\text { day }) & (\Delta t=10 \mathrm{~min}) \\
0.66 \times 10^{2} \text { (steps) } \simeq 0.92(\text { day }) & (\Delta t=20 \mathrm{~min})
\end{array}\right.
$$

and $\Delta t$ of $10 \sim 20 \mathrm{~min}$ involves fairly large dissipating effect.

Also, the leapfrog scheme is applied to the present calculation, in which Asselin time filter and initializations every 100 steps using substep forward differencing are required to warrant stable numerical integrations. For the same oscillation equation, amplification factor is given as

$$
\lambda^{\Delta t}=i \omega \Delta t \pm \sqrt{1-(\omega \Delta t)^{2}} .
$$

This scheme is neutral, i.e. $\left|\lambda^{\Delta t}\right|=1$ so long as $\omega \Delta t<1$. A minus sign corresponds to the computational mode, and initialization may reduce this mode relative to the physical mode (HALTINER 
and Williams, 1980). Backward implicit scheme was also tested to deal with diffusion terms, but is not used in the present calculations.

The numerical procedure is as follows. First, atmospheric and numerical model parameters and time-independent coefficients are given and estimated, and initial values for $\tilde{u}, \tilde{v}, \tilde{T}$ and $\tilde{z}(x=0)$ are specified. For each time step, we calculate forcing function and then solve the continuity equation downward for $\tilde{w}$ with the top value given by the simultaneous solution with the upper boundary condition. Hydrostatic equation is solved upward for $\tilde{z}$. Then, time derivative equations of the momentum, thermodynamic and lower boundary condition for $\tilde{u}, \tilde{v}, \tilde{T}$ and $\tilde{z}$ $(x=0)$ respectively, are calculated to update these quantities, and is repeated until prescribed time steps are reached.

We use a staggered grid in the altitude discretization in which $w, z$ are defined at the sub-grid point replaced by half the grid spacing from the grid point where $u, v$ and $T$ are defined. This leads to stability in numerical integration. Thus the continuity equation takes the form, after discretization,

$$
\tilde{w}_{i-1}=\frac{1-\frac{\Delta x}{4}}{1+\frac{\Delta x}{4}} \tilde{w}_{i}+\frac{\Delta x}{1+\frac{1}{4} \Delta x}\left(-\frac{\partial \tilde{u}}{\partial y}+\frac{1}{1-y^{2}} \frac{\partial \tilde{v}}{\partial \phi}\right)_{i} .
$$

\subsection{Thermotidal forcing}

In the present calculations, thermotidal forcing of each fundamental Hough mode is assumed for ozone and water vapor insolation absorption to investigate transient behavior of particular modes. A non-dimensionalized forcing $\tilde{Q}$ takes the form

$$
\hat{Q}^{\prime}(x, y, \hat{t})=\frac{R}{2 a g}\left[\tau_{G}^{\omega, 1}(x, y) e^{i(\hat{t} / 2-\pi)} e^{j \phi}+2 \tau_{G}^{2 \omega, 2}(x, y) e^{j 2(\hat{t} / 2-\pi)} e^{j 2 \phi}\right]
$$

Zero phase corresponds to local noon. $\tau_{G}^{\sigma, s}$ ( $\sigma$ : angular frequency) is the hypothetical temperature oscillation due to insolation absorption by a gas species $G$ which is in turn expressed by the product of latitudinal structure $g_{G}^{\sigma, s}$ and the prescribed altitude distribution $f_{G}^{\sigma, s}$ (CHAPMAN and LINDZEN, 1970).

$$
\left.\begin{array}{l}
\tau_{G}^{\omega, 1}=f_{H_{2} O}^{\omega, 1}(x) g_{H_{2} O}^{\omega, 1}(y)+f_{O_{3}}^{\omega, 1}(x) g_{O_{3}}^{\omega, 1}(y), \\
\tau_{G}^{2 \omega, 2}=f_{H_{2} O}^{2 \omega, 2}(x) g_{H_{2} O}^{2 \omega, 2}(y)+f_{O_{3}}^{2 \omega, 2}(x) g_{O_{3}}^{2 \omega, 2}(y) .
\end{array}\right\}
$$

The altitude distributions for both of the diurnal and semidiurnal forcings $f_{\mathrm{H}_{2} \mathrm{O}}$ and $f_{\mathrm{O}_{3}}$ are shown in Fig. 2(d).

$$
\begin{aligned}
f_{O_{3}} & = \begin{cases}\exp \left[0.0116\left(z-z_{1}\right)\right] \sin \frac{\pi\left(z-z_{1}\right)}{z_{2}-z_{1}} & \left(z_{1} \leq z \leq z_{2}\right) \\
0 & \left(z<z_{1}, z_{2}<z\right),\end{cases} \\
f_{H_{2} O} & = \begin{cases}\sin \frac{\pi\left(z-z_{4}\right)}{z_{3}-z_{4}} & \left(z_{4} \leq z \leq z_{3}\right) \\
0 & \left(z<z_{4}, z_{3}<z\right)\end{cases}
\end{aligned}
$$

where, $z_{1}=18 \mathrm{~km}, z_{2}=68 \mathrm{~km}, z_{3}=20 \mathrm{~km}, z_{4}=-12 \mathrm{~km}$. We confine forcings to classical modes of diurnal $(1,1),(1,-2)$ and semidiurnal $(2,2),(2,3),(2,4)$ modes as in ChAPMAN and LiNDZEN (1970) and LINDZEN and HONG (1974),

$$
\left.\begin{array}{rl}
g_{O_{3}}^{\omega, 1} & =1.6308 \Theta_{-2}^{\omega, 1}+0.5447 \Theta_{1}^{\omega, 1} \\
g_{H_{2},}^{\omega, 1} & =0.157 \Theta_{-2}^{\omega, 1}+0.062 \Theta_{1}^{\omega, 1} \\
g_{O_{3}, 2}^{2 \omega} & =0.249 \Theta_{2}^{2 \omega, 2}-0.0996 \Theta_{3}^{2 \omega, 2}+0.0645 \Theta_{4}^{2 \omega, 2} \\
g_{H_{2} O}^{2 \omega, 2} & =0.0307 \Theta_{2}^{2 \omega, 2}-0.0123 \Theta_{3}^{2 \omega, 2}+0.00796 \Theta_{4}^{2 \omega, 2} .
\end{array}\right\}
$$


(a)

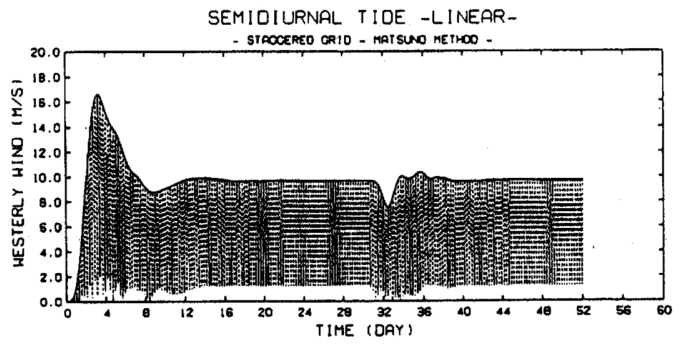

(b)

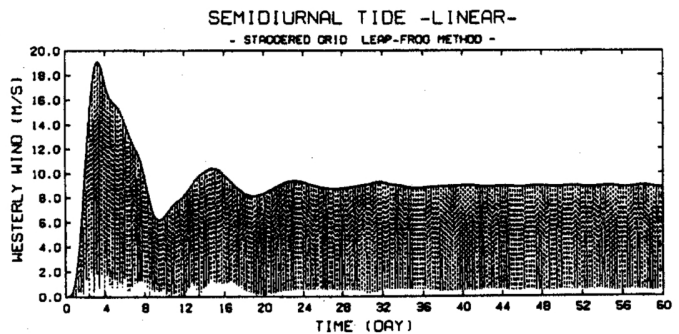

Fig. 3. Rise-up of the semidiurnal $(2,2)$ westerly wind component at $85.2 \mathrm{~km}, 30.5^{\circ} \mathrm{N}$ by the (a) Matsuno (MA) and (b) leap-frog (LF) methods.

The envelope for the forcing takes the form

$$
\frac{1}{2}\left\{1-\cos \left(\frac{\pi}{2 \omega T_{r}} \hat{t}\right)\right\}
$$

where $T_{r}$ is the rise time of forcing which is mostly 2 days unless otherwise noted.

\section{Result of Linearized Calculation}

In this section, time-dependent calculations for the linearized regime will be presented to quantitatively describe the aspects of tidal transience due to the onset of solar thermal forcing.

\subsection{Semidiurnal tide}

\section{Basic Characteristics}

For the semidiurnal tidal mode, vertical resolution was set to $\Delta x=0.3$ or 65 layers in the altitude. The maximum value of the degree of Legendre polynomial $l_{\max }$ is 9 . Calculations with $l_{\text {max }}=19$ do not alter the result for the semidiurnal dominant modes. Figure 3(a) shows the rise-up of semidiurnal $(2,2)$ westerly wind component at $85.2 \mathrm{~km}, 30.5^{\circ} \mathrm{N}$ by the Matsuno (hereafter referred to as the MA) method. It reaches its maximum at 3.3 days after the onset of forcing, accompanied by fairly small amplitude oscillation with increasing period of a few to several days. Also a sinusoidal reduction of forcing by $20 \%$ from steady values imposed during 30 to 32 th day gives rise to proportionate amplitude decrease followed by transient fluctuation for several days or more. Time step $\Delta t$ has an effect on artificial damping in the MA method. In the present calculation, $\Delta t$ is set to $5 \mathrm{~min}$. When $\Delta t$ is increased to $10 \mathrm{~min}$ to $20 \mathrm{~min}$, overshoot amplitude maximum reached at about 3 days after the onset reduces to about 80 and $60 \%$, respectively, relative to the result for $\Delta t=5 \mathrm{~min}$. When $\Delta t$ is set to $2.5 \mathrm{~min}$, amplitude 
maximum increases by $10 \%$. A steady state value for each $\Delta t$ is about $10 \mathrm{~m} / \mathrm{sec}$ and does not differ too much. This is reasonably compared with dissipation-free classical result for the Wilkes equation of about $13 \mathrm{~m} / \mathrm{sec}$ with the same forcing and temperature profile. It is suggested that the artificial damping which surpasses assumed dissipation parameters at particular heights works well to suppress higher frequeney gravity wave oscillations and to stabilize numerical calculations. As shown in Eq. (22), dissipation time constant for $\Delta t=5$ min time step is about 3.6 days for the semidiurnal oscillation period. Maximum value of $\alpha_{i}$ in Fig. 2(c) corresponds to e-folding time of about 2 days, while eddy diffusion coefficient of $40 \mathrm{~m}^{2} / \mathrm{sec}$ in Fig. 2(b) signifies roughly 8 days for a component with vertical wavelength of $50 \mathrm{~km}$. So, inherent dissipation associated with this scheme gives evolution of semidiurnal tide in the rather dissipative atmosphere.

Figure 3(b) shows the corresponding result calculated by the leap-frog (hereafter referred to as the LF) scheme. In this calculation, $l_{\max }=11$ and horizontal diffusion is introduced to avoid spurious growth of amplitude at higher latitudes due to wavenumber truncation. The horizontal diffusion term gives

$$
\left.\frac{\mu}{\rho} \nabla^{2} u\right|_{l, s}=\frac{\mu}{\rho a^{2}}\{-l(l+1)\} U_{l, s} .
$$

We choose $\mu / \rho \sim 10^{6} \mathrm{~m}^{2} / \mathrm{sec}$, so for $l=10$ and 16 component, dissipating time constant is 4.3 and 1.7 days, respectively, which is appropriate to attenuate higher order modes, and is considered to be an acceptable parameterization which tidal waves encounter in the middle atmosphere. In the figure, amplitude likewise reaches its maximum value at 3.3 days after the onset, but the amplitude oscillation with period of about $8 \sim 10$ days are evident. Horizontal diffusion of $10^{7} \mathrm{~m}^{2} / \mathrm{sec}$ gives more damped amplitude evolution of $(2,2)$ mode and is very similar to the MA result in Fig. 3(a). So in the LF scheme, $10^{6} \mathrm{~m}^{2} / \mathrm{sec}$ is hereafter assumed in the calculation to follow, to look into less dissipative tidal evolution. Also, the time step $\Delta t$ has some effect in a less pronounced way on the LF result.

Figures 4(a) and (b) show latitude-time contours of semidiurnal $(2,2)$ westerly wind at 85.2 $\mathrm{km}$ for $0 \sim 30$ th day by the MA and LF methods, respectively. Latitudinal structure corresponding to the velocity expansion function of $(2,2)$ westerly wind is seen with maximum at around $30^{\circ} \mathrm{N}$. At lower latitudes for the LF result, fluctuations in complex amplitude with period of about 0.6 day is observed. If rise-time of forcing $T_{r}$ is chosen to be 1 day, wave field evolves faster with the peak of westerly wind at $30^{\circ} \mathrm{N}$ at 2.6 days after the onset. When $T_{r}$ is set to 0.5 day, its peak occurs at 2.4 days but more well-defined ripples in complex amplitude are superposed at all latitudes with similar period of about 0.6 day. For $T_{r}=4$ day, it rises gradually, but in all cases, amplitude oscillation with period of about $8 \sim 10$ days appears irrespective of $T_{r}$.

Vertical propagation

Figure 5 shows the result of vertical propagation of $(2,2)$ mode westerly wind component at $30.5^{\circ} \mathrm{N}$ excited only by water vapor calculated by the LF method. The wind value is normalized by its peak value at each altitude. In the figure, it is seen that the first maximum amplitude excited by water vapor below $20 \mathrm{~km}$ propagates upward with vertical group velocity of roughly 0.6 $\mathrm{m} / \mathrm{sec}$. As for the $(2,4)$ mode, Fig. 6 shows the vertical propagation of westerly wind component at $63.7^{\circ} \mathrm{N}$ by the LF method. Westerly wind amplitude at this latitude is larger than at $30.5^{\circ} \mathrm{N}$ for the $(2,4)$ mode. It is indicated that amplitude oscillation is weak, and vertical group velocity is on the order of $0.4 \mathrm{~m} / \mathrm{sec}$, which is relatively smaller than $(2,2)$ mode velocity.

Time evolution and steady tidal structure

Figure 7 shows time evolution of $(2,2)$ mode tidal structures at $30.5^{\circ} \mathrm{N}$ for the westerly wind component at 1-10th day after onset. This result only is calculated by the vertical resolution of $\Delta x=0.1$. These are altitude profiles of amplitude and phase. Amplitude and phase are the absolute value and phase of particular complex wave field at the specified time assuming sinusoidal oscillation of the form $A \varepsilon^{j(\omega t-\phi)}$. It is seen that amplitude grows fast and phase profiles indicate 
(a)

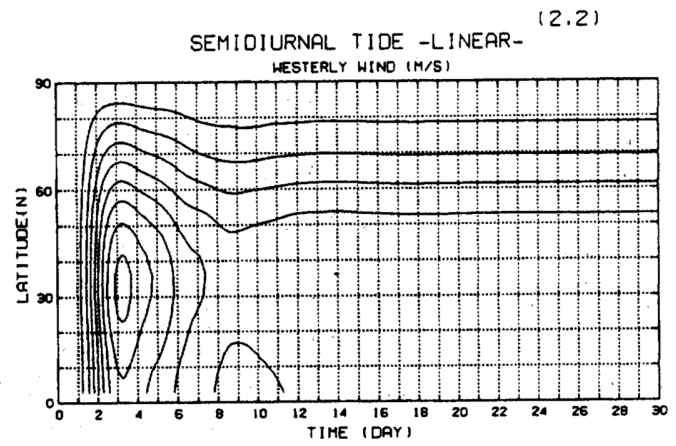

(b)

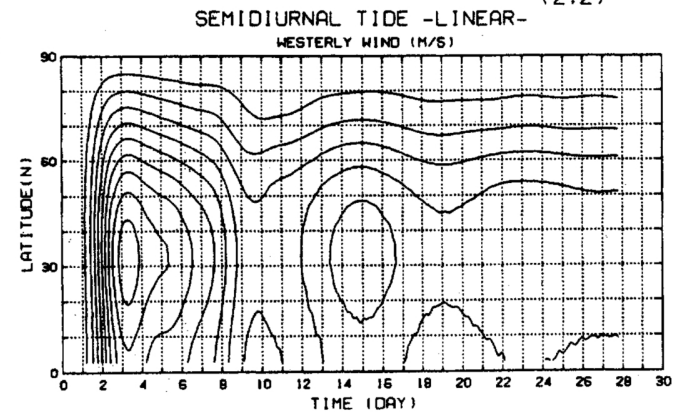

Fig. 4. Latitude-time contour of semidiurnal $(2,2)$ westerly wind at $85.2 \mathrm{~km}$ by the (a) MA and (b) LF methods. Contour interval is $2 \mathrm{~m} / \mathrm{sec}$.

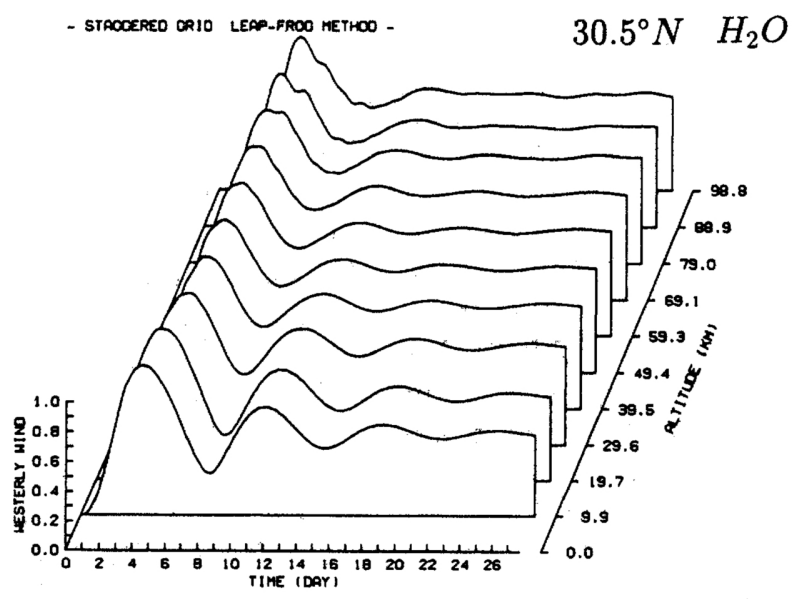

Fig. 5. Vertical propagation of $(2,2)$ mode excited only by water vapor calculated by the LF method. 


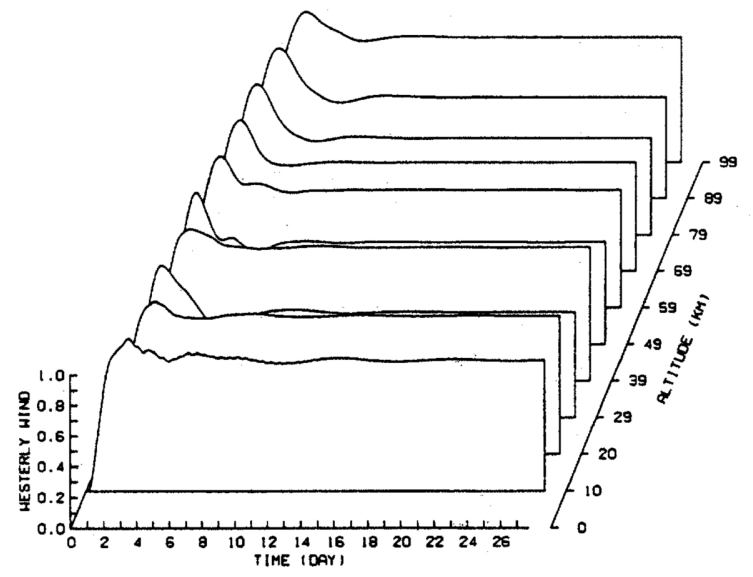

Fig. 6. Vertical propagation of $(2,4)$ mode westerly wind component at $63.7^{\circ} \mathrm{N}$ by the LF method.
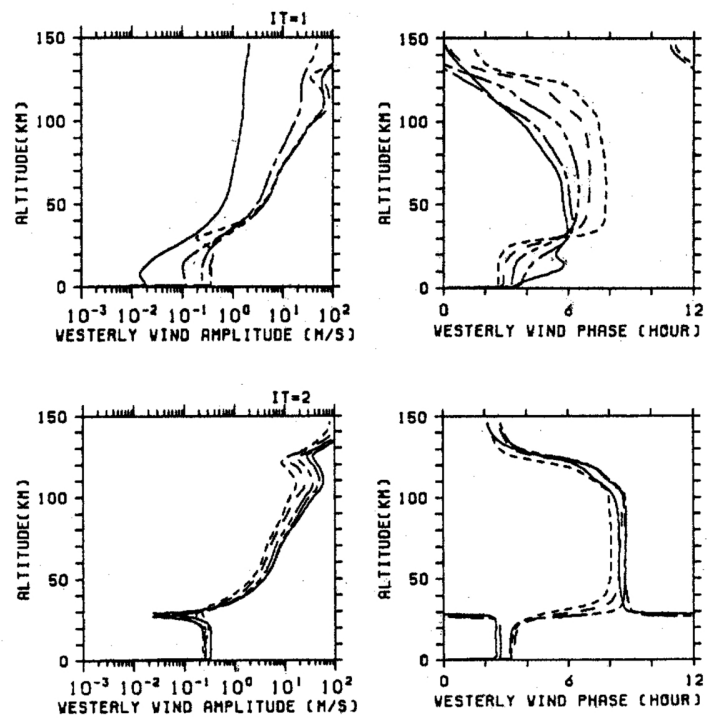

Fig. 7. Time evolution of $(2,2)$ mode tidal structures at $30.5^{\circ} \mathrm{N}$ for the westerly wind component at 1-5th (above) and 6-10th (below) day after onset. Lines are 1 or 6 (solid line), 2 or 7 (dot-dashed line), 3 or 8 (dashed-double dotted line), 4 or 9 (dashed line) and 5 or 10 (dotted line) th day. 

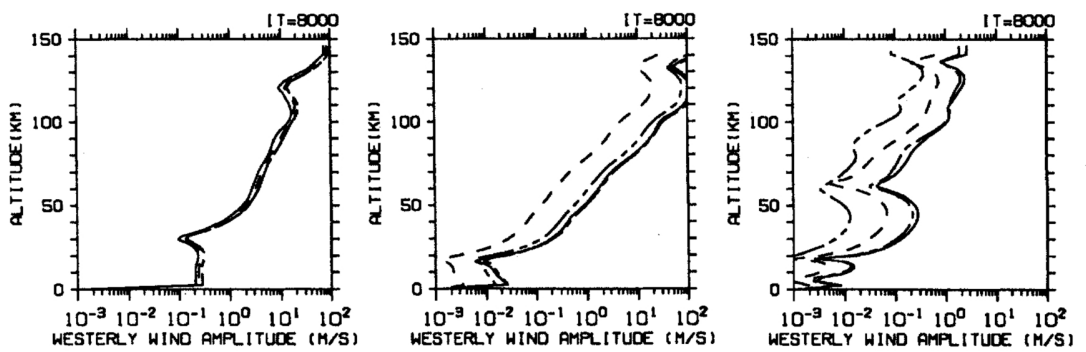

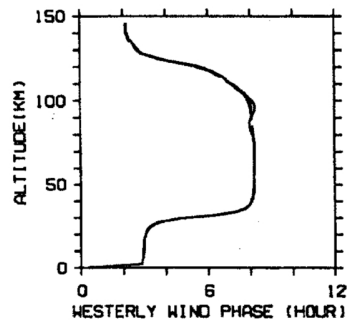

$(2,2)$

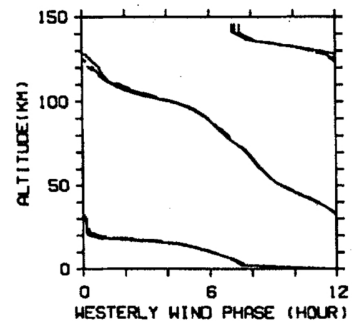

$(2,3)$

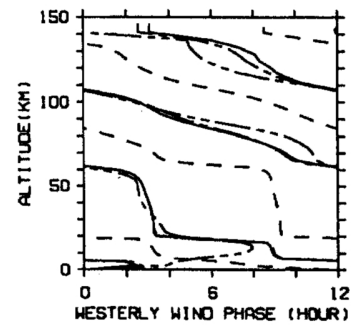

$(2,4)$

Fig. 8. Steady state tidal structures of the westerly wind component for $(2,2),(2,3)$ and $(2,4)$ modes at 27.8 th day after onset of forcing. Latitudes are $58.1^{\circ} \mathrm{N}$ (solid line), $41.5^{\circ} \mathrm{N}$ (dot-dashed line), $24.9^{\circ} \mathrm{N}$ (dashed-double dotted line) and $8.3^{\circ} \mathrm{N}$ (dashed line).

shorter vertical wavelength for the first few days and tend to be steady structure, viz., propagating above $100 \mathrm{~km}$, and evanescent in the middle atmosphere with phase reversal at around $30 \mathrm{~km}$ which reflects interference of tides excited respectively by ozone and water vapor forcings.

In Fig. 8 is shown steady structures of $(2,2),(2,3)$ and $(2,4)$ modes at 27.8 th day after the onset of forcing for the westerly wind component at four latitudes $58.1,41.5,24.9,8.3^{\circ} \mathrm{N}$. Altitude profile of northerly wind component is very similar to the westerly one except for its phase lag of 3 hours. The results are fairly compatible with those calculated by the linearized steady modelling or by the simple classical tidal theory especially at lower altitudes where dissipating effect can be neglected.

\subsection{Diurnal Tide}

\section{Evanescent $(1,-2)$ mode}

To calculate the evolution of evanescent $(1,-2)$ mode which has almost constant phase structure, vertical resolution was likewise set to be $\Delta x=0.3$ as in the semidiurnal mode. The maximum degree of Legendre expansion $l_{\max }$ is also 16. Figure 9 shows the evolution of westerly wind component at $63.7^{\circ} \mathrm{N}$ calculated by the (a) MA and (b) LF method. In the figure, amplitude at each height is normalized as in Fig. 5. It is seen that rise time is fairly small, about 1 day and no altitude variation of time delay or phase change is evident. This shows how the evanescent mode builds up in the altitude direction. Also, beat in complex amplitude with period of about $1.2 \sim 1.3$ day is clearly seen. As will be mentioned in the discussion, this might be considered to be due to the existence of both diurnal oscillation and $5 \sim 7$-day $(0.15 \sim 0.2 \mathrm{c} /$ day $)$ normal mode of wavenumber 1 excited possibly by the transient forcing of diurnal tidal oscillation.

Time evolution of altitude profile of $(1,-2)$ mode at $58.1^{\circ} \mathrm{N}$ is shown in Fig. 10 for the westerly wind component from 1 to 10 th day as in Fig. 7 . Below $90 \mathrm{~km}$, amplitude reaches its steady-state profile rather fast, and almost constant phase characteristics of evanescent mode is 

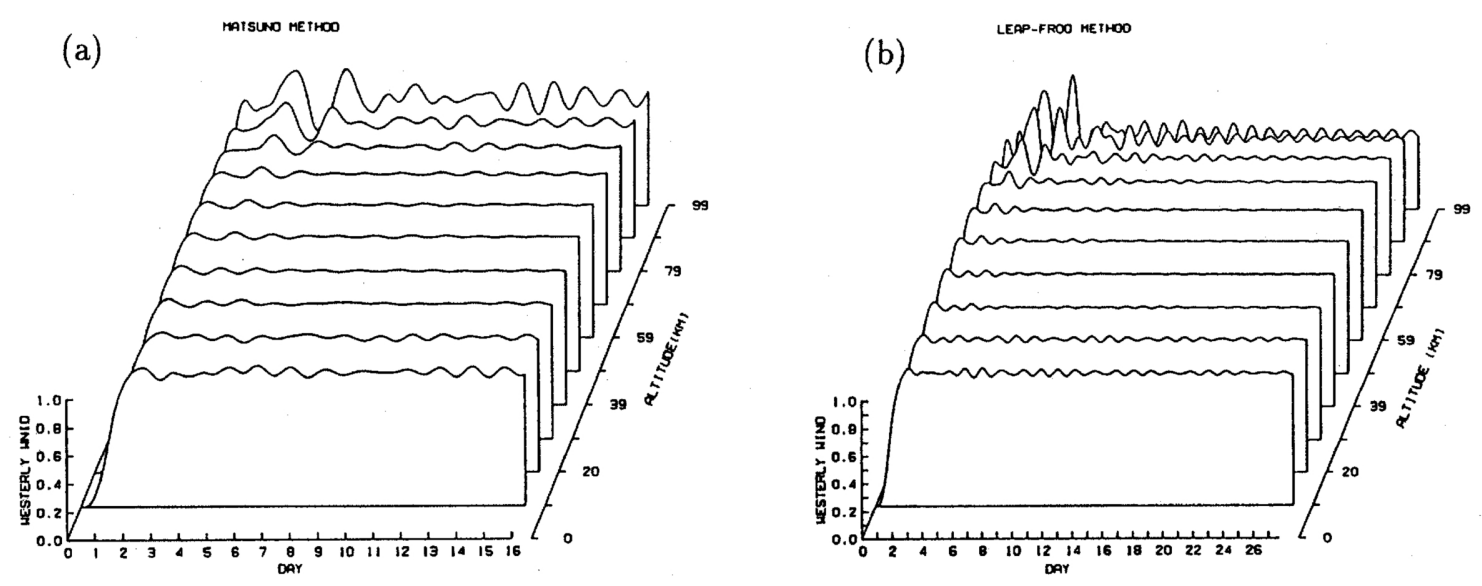

Fig. 9. Evolution of diurnal $(1,-2)$ westerly wind component at $63.7^{\circ} \mathrm{N}$ calculated by the (a) MA and (b) LF method.
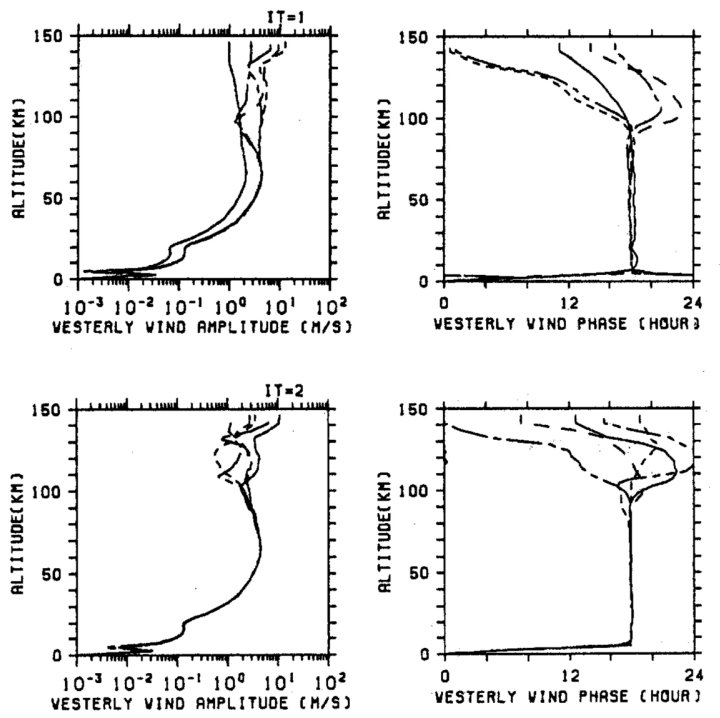

Fig. 10. As in Fig. 7 except for $(1,-2)$ mode at $58.1^{\circ} \mathrm{N}$. 
(a)
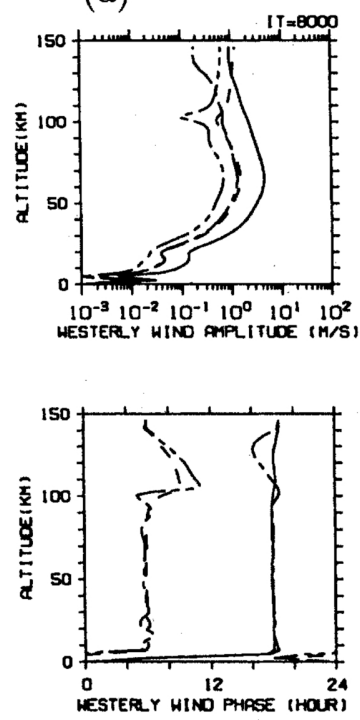

(b)
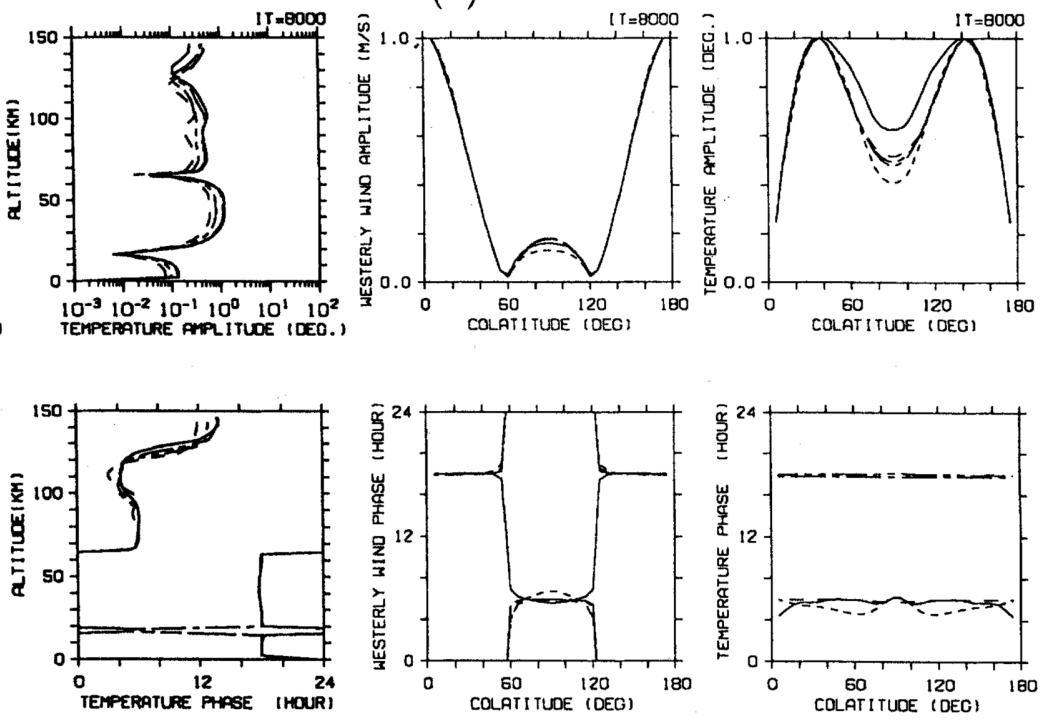

Fig. 11. Steady state (a) altitude and (b) latitudinal structures of diurnal $(1,-2)$ westerly wind velocity and temperature perturbations reached after 27.8 days after the onset of forcing. In (a), altitudes are 18.3 (solid line), 38.1 (dot-dashed line), 61.4 (dashed-double dotted line), 80.0 (dashed line) and 97.1 (dotted line) km. Latitudes in (b) are as in Fig. 8.

established in an early stage. On the other hand, above $90 \mathrm{~km},(1,-2)$ mode tends to be of propagating mode in the dissipative atmosphere, and erratic phase variation is seen to occur, corresponding to enhanced oscillation as in Fig. 9.

Steady state structures reached at 27.8th day after the onset of forcing are shown in Fig. 11 as (a) altitude and (b) latitudinal structures of westerly velocity and temperature perturbations. The results are again consistent with known structures of $(1,-2)$ mode revealed by the classical theory or linearized steady calculations in the dissipative atmosphere.

\section{Propagating $(1,1)$ mode}

Propagating $(1,1)$ mode indicates rapid phase excursion with altitude, and resolution of $\Delta x=0.1$ is required to fully describe the mode. $l_{\max }$ here is 19 . Figure $12(\mathrm{a})$ is a latitudinal contour plot of westerly wind component at $85.2 \mathrm{~km}$ and amplitude growth at $30.5^{\circ} \mathrm{N}$ is shown in Fig. 12(b). The forcing is water vapor and ozone. The scheme is the LF method and gives almost identical result with the MA method which, as mentioned before, involves longer e-folding time of 15 days for the time step of $\Delta t=5 \mathrm{~min}$ for the diurnal oscillation and inherent dissipation is not crucial. Latitudinal structure conforms to the velocity expansion function of $(1,1)$ mode which has its maximum just equatorward of $30^{\circ}$. Apart from small forerunner at several days after the onset, peak amplitude is attained at about 10th day or later which indicates smaller vertical group velocity for the setup of tidal structure.

Figure 13 shows vertical propagation of $(1,1)$ mode more explicitly. In this case, water vapor of smaller vertical extent seems to excite the $(1,1)$ mode more efficiently and peak amplitude propagates upward in rather well-defined way. The vertical group velocity indicated in Fig. 13 is about $0.1 \mathrm{~m} / \mathrm{sec}$.

The evolution of vertical and latitudinal structures of $(1,1)$ mode westerly components at $30.5^{\circ} \mathrm{N}$ and at $81.2 \mathrm{~km}$, respectively are shown in Figs. 14(a) and (b). In the course of evolution, 

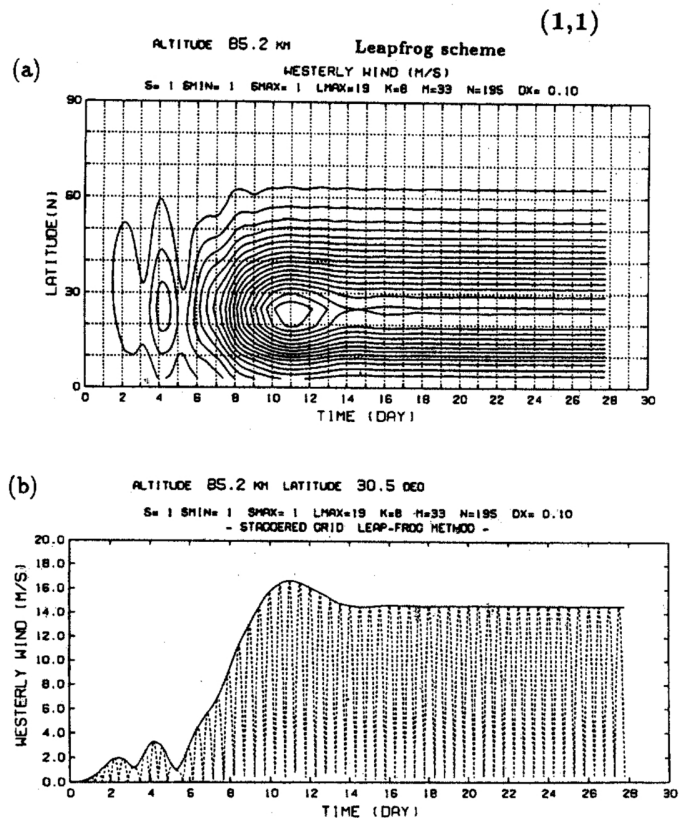

Fig. 12. (a) Latitudinal contour plot and (b) amplitude growth at $30.5^{\circ} \mathrm{N}$ of diurnal $(1,1)$ westerly wind component at $85.2 \mathrm{~km}$. Contour interval is $1 \mathrm{~m} / \mathrm{sec}$.

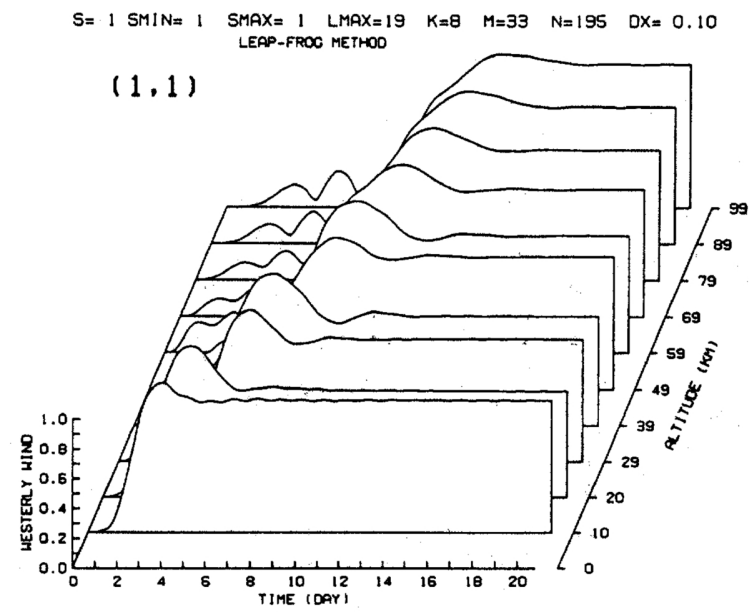

Fig. 13. Vertical propagation of $(1,1)$ mode at $30.5^{\circ} \mathrm{N}$. 
(a)
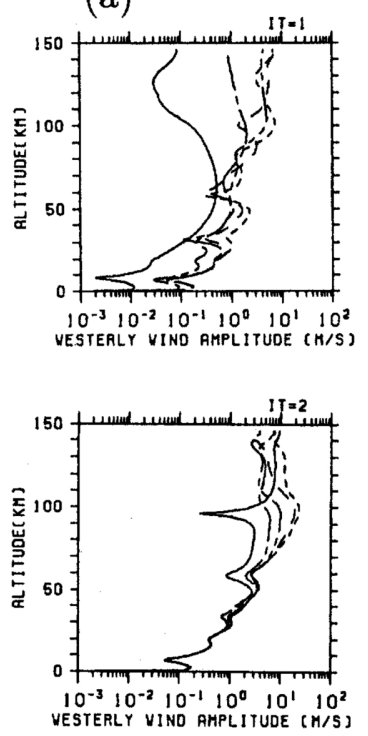
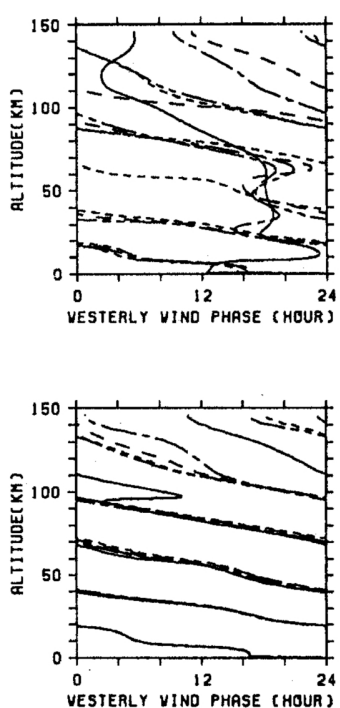

(b)
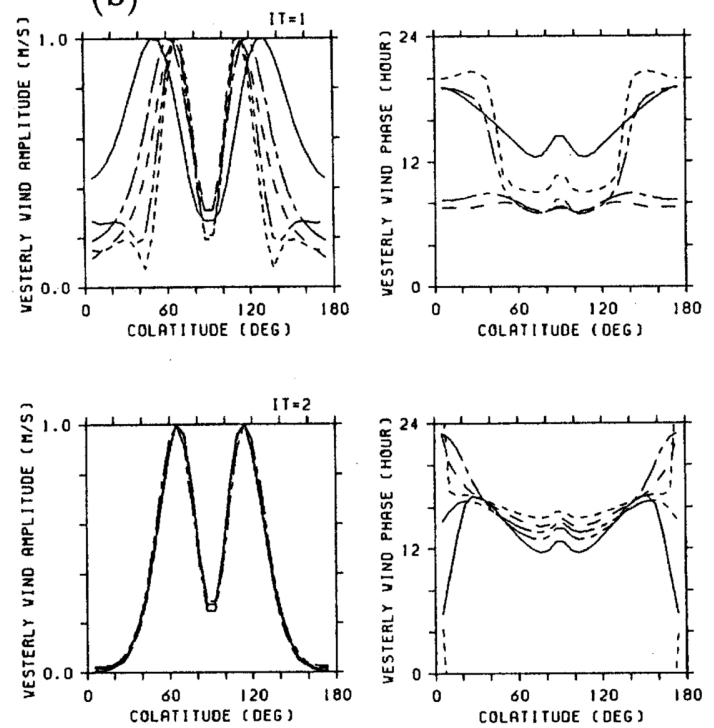

Fig. 14. Evolution of (a) vertical and (b) latitudinal structures of diurnal $(1,1)$ mode westerly components. Lines are as in Fig. 7.

amplitude grows and phase structure tends to be of short vertical wavelength from fairly constant feature in the forcing region. At 5-6th day, phase structure below $100 \mathrm{~km}$ is close to its steadystate short vertical wavelength characteristics, and above $100 \mathrm{~km}$, it becomes stable later on after about 10th day. Latitudinal structure correspondingly indicates extinction of mixed evanescenttype behavior which prevails at mid to high latitude. Rapid phase excursion near poles where amplitude is fairly small after 6th day may be an artifact due to polynomial truncation.

\section{Results of Nonlinear Calculation}

In the preceding calculations, nonlinear effects have been neglected. It is, however, possible that the contribution of nonlinear terms becomes significant as the amplitude becomes large in the higher altitude and comparable to linear terms in the primitive equation system. Here, we carry out nonlinear calculations, assuming only two zonal wavenumber components $s=1$ and $s=2$ to quantitatively estimate the effect of mode coupling and, accordingly, the excitation of semidiurnal components by the diurnal thermotidal forcing. We consider the nonlinear terms indicated by braces in Eqs. (8)-(10). Also, the retention of the term $\partial \hat{z}_{1} / \partial x$ in the boundary condition of Eq. (19) is required. Forcing of diurnal migrating wave, i.e., $s=1$ and 24 hour period oscillation drives diurnal migrating tidal wave and also $s=2$ harmonic component as well, its behavior determined by a so called "harmonic balance" sense. In the following calculation, the LF method is used with $l_{\max }=18$ and $\Delta x=0.1$ throughout.

Figure 15 shows nonlinear time evolution of westerly wind component at $85.2 \mathrm{~km}$ height and $30.5^{\circ} \mathrm{N}$ excited by the diurnal $(1,1)$ mode ozone and water vapor forcings with $s=1$ and 2 put together. A rise up characteristic is very similar to the diurnal linear calculation with $s=1$ given in Fig. 12(b). The only difference is the superimposed amplitude modulation with a period of just one day which appears after about 6 th day. This is caused by the beat of diurnal component with upcoming excited semidiurnal component. 


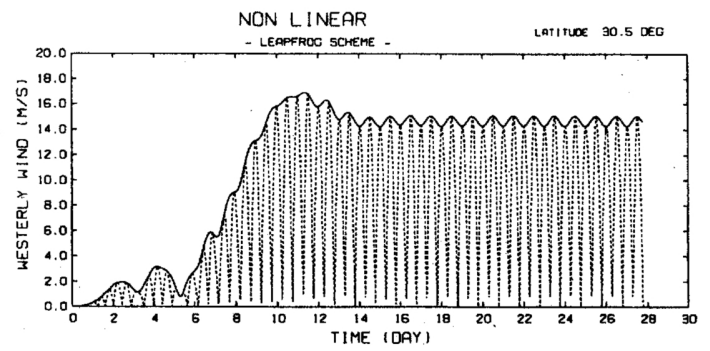

Fig. 15. Nonlinear time evolution of diurnal westerly wind component at $85.2 \mathrm{~km}$ height and $30.5^{\circ} \mathrm{N}$ excited by $(1,1)$ mode ozone and water vapor forcings.

(a)

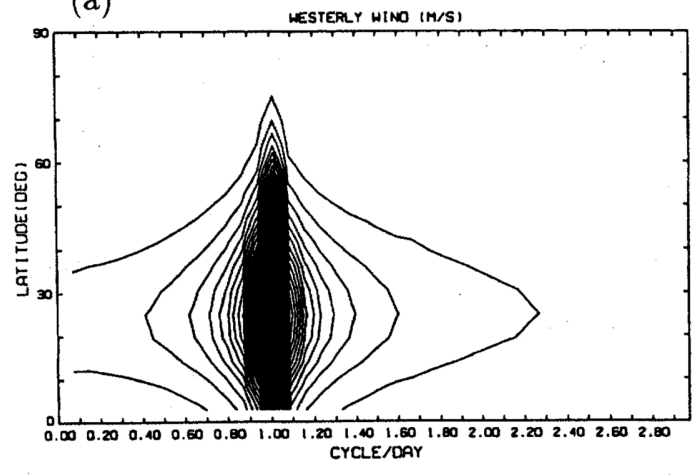

(b)

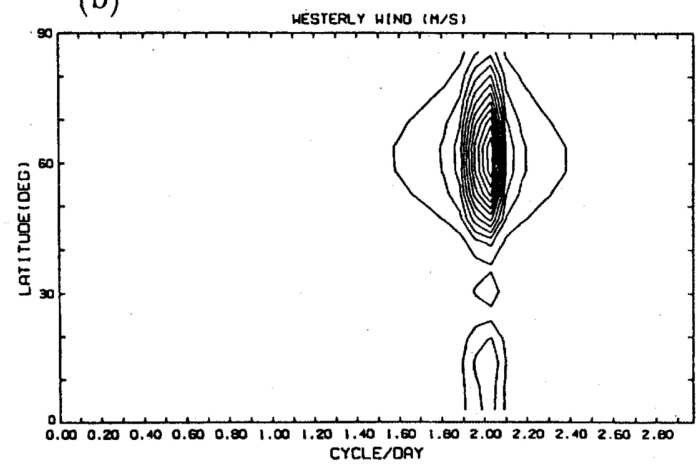

Fig. 16. Latitudinal contour of the spectrum analysis for the (a) $s=1$ and (b) $s=2$ components excited by the diurnal $(1,1)$ mode ozone and water vapor forcings.

Figure 16 shows latitudinal contour plot for the spectrum analysis of the westerly wind for the $s=1$ and $s=2$ components at $85.2 \mathrm{~km}$. A period of analysis is from 14 th to $28 \mathrm{th}$ day from the onset. It is quite natural that $s=1$ component has a dominant period of one day and has its peak value at $30^{\circ}$ corresponding to $(1,1)$ mode excitation. On the other hand, $s=2$ harmonic mode is predominantly semidiurnal, and therefore it is evident that the "migrating" semidiurnal component is excited. It has a peak at around $60^{\circ} \mathrm{N}$ and suggest the signature of higher order semidiurnal modes. Also, both peaks indicate some spread, suggesting notable fluctuations in its frequency.

Figure 17 shows latitudinal contour plot of the evolution of (a) $s=1$ and (b) $s=2$ of the westerly wind at $85.2 \mathrm{~km}$ excited by the $s=1$ diurnal $(1,1)+(1,-2)$ ozone and water vapor forcings. Contour interval is $1 \mathrm{~m} / \mathrm{sec}$. A contour plot for $s=1$ is very similar to the linear calculation and indicates maximum around $30^{\circ}$ and $90^{\circ} \mathrm{N}$ corresponding respectively to $(1,1)$ and $(1,-2)$ velocity expansion functions. $(1,1)$ mode peak appears around 11th day as in Fig. 12. Also, complex amplitude indicates a beat with a period of about 1.2 days associated with $(1,-2)$ forcing. On the other hand, $s=2$ component has maximum value of about $4 \mathrm{~m} / \mathrm{sec}$ at around $60^{\circ} \mathrm{N}$ at 9 th day. This may corresponds to the rather slow evolution of diurnal $(1,1)$ mode. For $(1,-2)$ mode forcing not shown here, excited $s=2$ westerly wind component at 85.2 $\mathrm{km}$ reaches its peak of some $1 \mathrm{~m} / \mathrm{sec}$ at $50^{\circ} \mathrm{N}$ at 6 th day from the onset, whereas $s=1$ westerly wind component peak is about $6 \mathrm{~m} / \mathrm{sec}$ near the pole.

Figure 18 shows non-linear evolution of $(1,1)$ mode forcing at $30.5^{\circ} \mathrm{N}$ in the time-height 
(a)

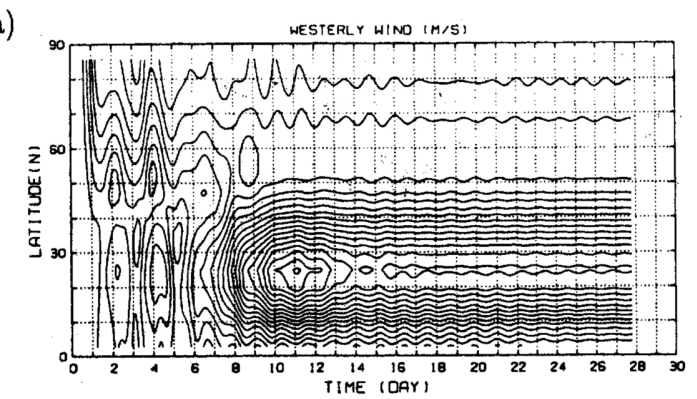

(b)

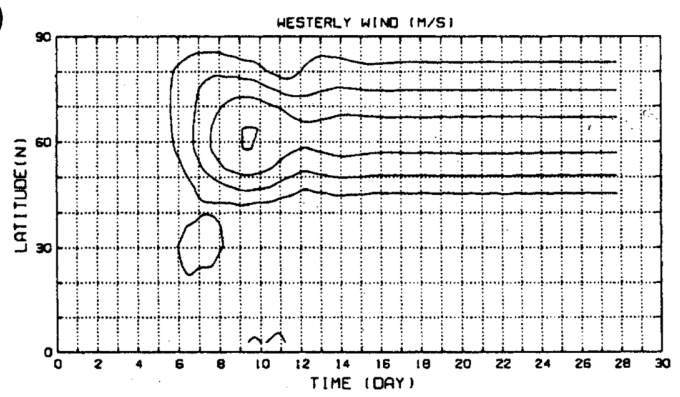

Fig. 17. Latitudinal contour plot of the evolution of (a) $s=1$ and (b) $s=2$ westerly wind component nonlinearly excited by the diurnal $(1,1)+(1,-2)$ ozone and water vapor forcings.

diagram which indicates superposition of excited $s=2$ component. The westerly wind components for (a) $s=1$ and (b) the composite of $s=1$ and $s=2$ are illustrated. In the figure, wind values are normalized to unity by its maximum value at each height and contour interval is 0.1 . In this case, the forcing is only water vapor below $20 \mathrm{~km}$ and upward propagation as in the linear regime is indicated in Fig. 18(a) with inferred vertical propagation velocity of about $0.1 \mathrm{~m} / \mathrm{sec}$. Effect of excited semidiurnal component is evident in Fig. 18(b) above about $60 \mathrm{~km}$ where amplitude becomes large enough to interact with diurnal component. This occurs after $7 \sim 8$ th day corresponding to upcoming of $s=2$ component above this height.

\section{Discussions}

\section{Rise time and vertical propagation}

From the linearized calculation, westerly wind component of tides at meteor heights excited in the lower atmosphere water vapor with a rise time of forcing of 2 days reaches its peak at around $80 \mathrm{~km}$ after about 3 days for $(2,2)$ and $(2,4)$ mode and about 11 days for $(1,1)$ mode. Time height diagram indicates the vertical propagation and the inferred vertical group velocity is $0.6 \mathrm{~m} / \mathrm{sec}$ for $(2,2)$ mode, $0.4 \mathrm{~m} / \mathrm{sec}$ for $(2,4)$ mode and $0.1 \mathrm{~m} / \mathrm{sec}$ for $(1,1)$ mode. VIAL et al. (1991) gives $1.4,0.3,0.12 \mathrm{~m} / \mathrm{sec}$ for $(2,2),(2,4)$ and $(1,1)$ modes, respectively. As in Vial et $a l$., vertical group velocity is calculated using the basic temperature profile in Fig. 1(a) and the table of dispersion characteristics given by RICHMOND (1975), and the values of $0.42-0.56 \mathrm{~m} / \mathrm{sec}$ for $(2,4)$ and $0.12-0.17 \mathrm{~m} / \mathrm{s}$ for $(1,1)$ are estimated. For $(2,2)$ mode, tide is basically evanescent in the mesosphere and values of $0.54-0.58 \mathrm{~m} / \mathrm{sec}$ are inferred in the lower altitude region. Hence the result seems to correspond to these theoretical values fairly well.

Also, set-up time defined by VIAL et al. (1991) as the time necessary to establish the sta-. tionarity of tide depends on the dissipation time constant as in Fig. 2 and dissipations inherent 
(a)

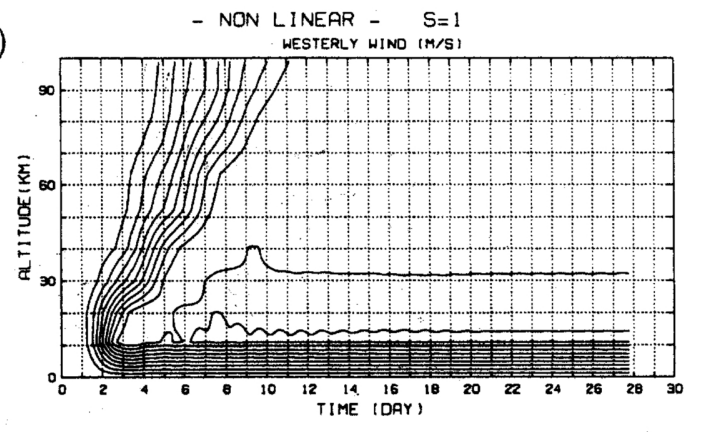

(b)

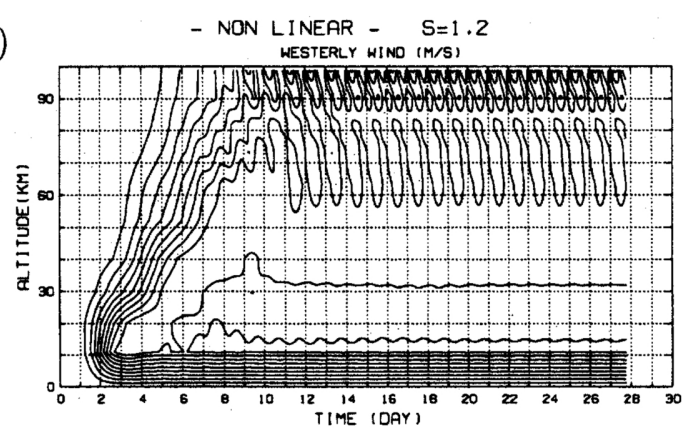

Fig. 18. Nonlinear time evolution of diurnal (a) $s=1$ component and (b) composite component superimposed by the $s=2$ component at $30.5^{\circ} \mathrm{N}$ excited by $(1,1)$ mode in the time-height diagram.

in the MA schemes, but the value is 20-40 days for $(2,2)$, and 15-20 days for $(1,1)$ which is, by and large, consistent with those given by VIAL et al. (1991) in their calculation at $75 \mathrm{~km}$ and $T_{r}$ $=50$ hours.

\section{Oscillation of complex amplitude}

Oscillation of complex amplitude is evident in the transience of tidal evolution. For $(2,2)$ mode, its period is $8-10$ days. This period is not sensitive to the rise time of forcing $T_{r}$. It might be associated with the normal mode $\varepsilon^{-\frac{1}{2}}=\sqrt{g h} / 2 \omega a \approx 0.34$ ( $\varepsilon$ : Lamb's parameter, $h$ : equivalent depth), very close to semidiurnal period, and has a beat of low frequency or long period. For the short period component with period of about 0.6 day observed especially at rapid rise-up of forcing, free wave normal mode of $s=2$ westward traveling mode which has frequency around $0.25 \mathrm{c} / \mathrm{d}$ might be a candidate to give a beat frequency of $1.75 \mathrm{c} / \mathrm{d}$ corresponding to that period.

As for the $(1,-2)$ mode, oscillation of complex amplitude with a period of 1.2 1.3 day is evident. If the $s=1$ normal mode planetary $5 \sim 7$ day wave $(0.15 \sim 0.2 \mathrm{c} /$ day $)$ is excited, complex amplitude reveals beat of $(1-0.15 \sim 0.2) \mathrm{c} /$ day $=0.8 \sim 0.85 \mathrm{c} /$ day or $1.2 \sim 1.25$ day period.

Figure 19 shows the frequency spectrum analysis versus latitude of the $(1,-2)$ westerly wind component. A peak around $0.15 \sim 0.2 \mathrm{c}$ /day is seen to exist at low and high latitudes, but is very small at around $40^{\circ} \mathrm{N}$. This characteristics is not incompatible with eigenfunction of westerly components of velocity of 5 7-day wave, a westward traveling class 2 normal mode wave of wavenumber $s=1$ (Longuet-HigGins, 1968). Another possible interpretation in terms of the interference of tides excited in respective altitude regions has been suggested by VIAL et al. (1991). Nonlinear excitation of semidiurnal waves

It has been shown that sinusoidal diurnal forcing excites semidiurnal oscillation of wavenumber 2 due to nonlinear characteristics of the governing equations. Figure 20(a) illustrates semidi- 


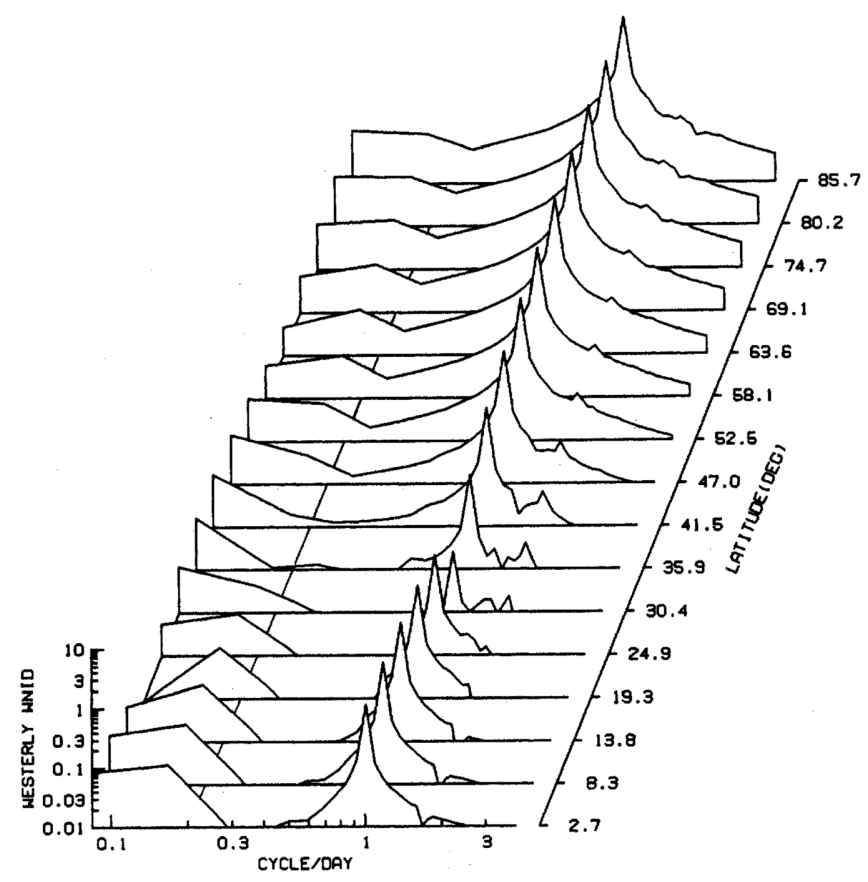

Fig. 19. Spectrum analysis versus latitude of the $(1,-2)$ westerly wind component at $85.2 \mathrm{~km}$.

(a)
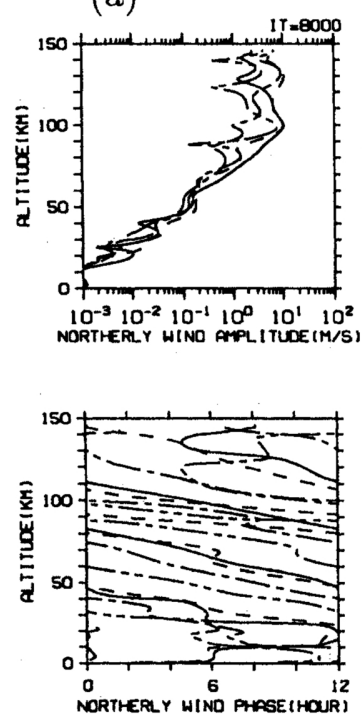

(b)
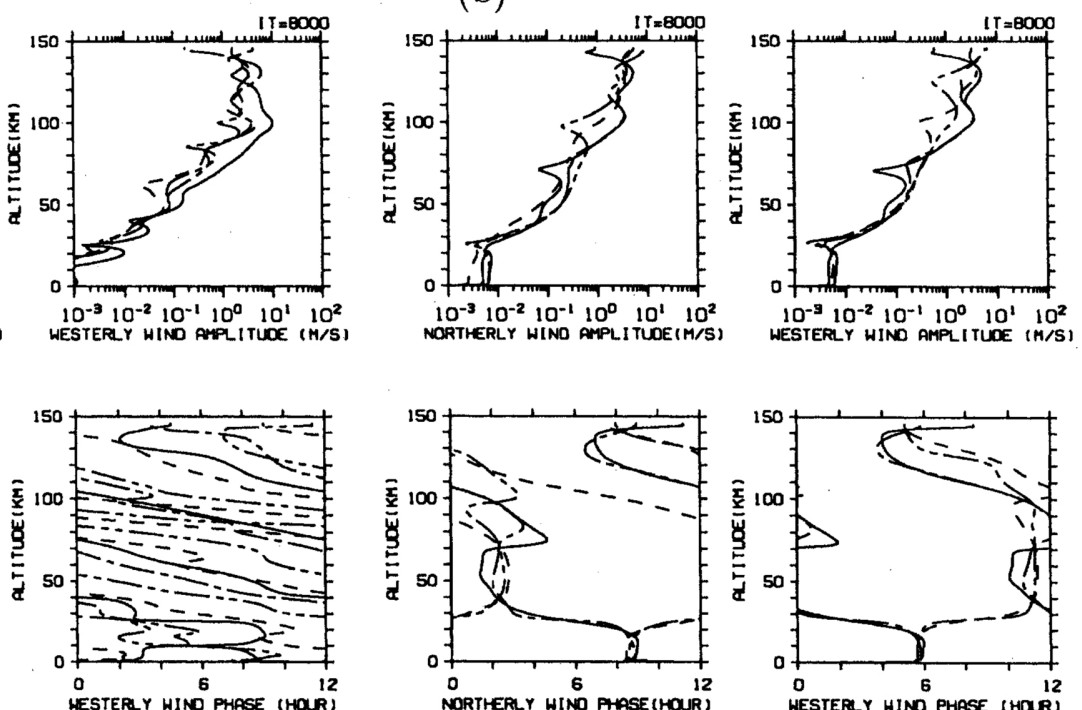
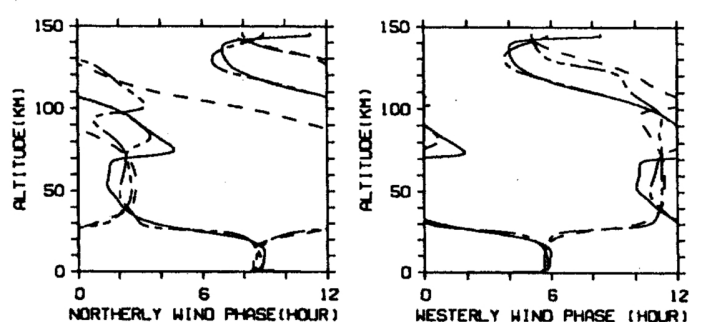

Fig. 20. Semidiurnal tide of northerly and westerly wind components nonlinearly excited by the diurnal (a) $(1,1)$ and (b) $(1,-2)$ mode forcings at 27.8 th day after onset. Latitudes are as in Fig. 8. 

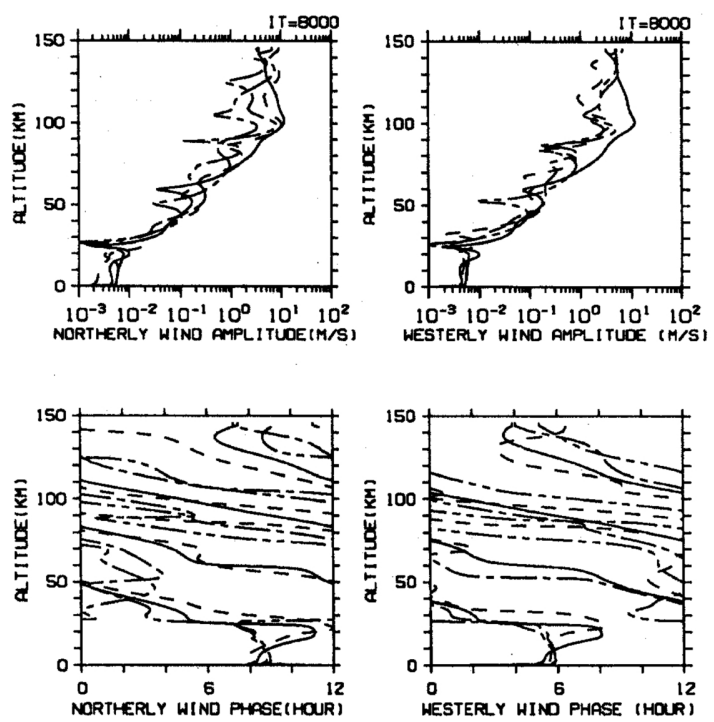

Fig. 21. As in Fig. 20 except for the $(1,1)$ and $(1,-2)$ mode forcings.

urnal northerly and westerly wind components excited by diurnal $(1,1)$ mode drive at 27.8 th day after the onset. It is clearly seen that amplitude grows with altitude roughly in proportion to the square of the $(1,1)$ mode growth and has an amplitude of several to $10 \mathrm{~m} / \mathrm{sec}$ at $90 \mathrm{~km}$ which is not negligibly small compared with the linearly excited semidiurnal components. Its vertical wavelength is rather short corresponding to $(1,1)$ mode compared to the dominant semidiurnal mode, and infers the existence of higher order modes which prevails at higher latitudes. Wu et al. (1989) also found in their simplified GCM calculations that diurnal $s=1$ heating excites semidiurnal component of some $10 \mathrm{~m} / \mathrm{sec}$ in which diurnal component itself is slightly larger than our result due to the difference of forcing and dissipation parameters.

On the other hand in Fig. 20(b), the semidiurnal component excited by the evanescent $(1,-2)$ forcing has amplitude of a few $\mathrm{m} / \mathrm{sec}$ and relatively long vertical wavelength corresponding to $(1,-2)$ mode. The phase variation is very similar to $(2,2)$ mode but lags by about 3 hours relative to $(2,2)$ phase.

In Fig. 21, semidiurnal component excited by $(1,1)$ and $(1,-2)$ forcing is shown. It is seen that evanescent structure at lower altitude is replaced by the short vertical wavelength propagating characteristics with rapid increase in amplitude. This reflects the existence of higher order short vertical wavelength component in the semidiurnal oscillation. It can be anticipated that the excited short vertical wavelength semidiurnal component may contribute to the variability of semidiurnal tidal field in the middle atmosphere due to variations of diurnal component of short vertical wavelength through the mode coupling described in this section.

\section{Conclusion}

A time-dependent spectral model of the nonlinear primitive equation is solved to quantitatively estimate the evolution of solar thermal tide in the middle atmosphere. Rise-up of semidiurnal $(2,2),(2,4)$ and diurnal $(1,1),(1,-2)$ modes is investigated and rise-time values at $85 \mathrm{~km}$ of about 3 days and 11 days are found for the semidiurnal and diurnal components, respectively, to 2 day rise time forcing. Vertical group velocity associated with this evolution is also estimated 
and found to be consistent with theoretical values. Oscillation of complex amplitude due to tidal transience has been detected. For $(2,2)$ mode, it has a long period of about 8 10 days and suggests the excitation of normal mode wave which has an eigenperiod very close to semidiurnal period. Also, short period beat associated with excitation of longer period normal mode is found for rapid-rise forcing. In the transience of $(1,-2)$ mode, excitation of $5 \sim 7$-day period normal mode wave is suggested, and latitudinal distribution of the spectrum component was compatible with the eigenfunction of zonal velocity component.

Non-linearities due to the advection terms of the primitive equation contribute to the excitation of semidiurnal component by the diurnal thermal drive. Diurnal $(1,1)$ forcings excite rather short vertical wavelength semidiurnal component with latitudinal maximum around $60^{\circ} \mathrm{N}$ , suggesting the excitation of higher order modes. Semidiurnal zonal or meridional wind at 90 $\mathrm{km}$ is several to $10 \mathrm{~m} / \mathrm{sec}$.

On the contrary, evanescent $(1,-2)$ mode forcing gives rather long vertical wavelength mode with maximum of westerly wind around $50^{\circ} \mathrm{N}$, and slightly smaller amplitude. This nonlinearly excited wave component contributes to the variability of otherwise stable semidiurnal tide due to variations of linearly driven diurnal component.

Further development of the present time-dependent calculation should numerically manifest upward group propagation and possible interaction with other planetary-scale winds and waves, and also contribution of non-migrating tidal forcings. These findings might eventually give quantitative information to delineate evolution and variable characteristics of solar thermal tide observed in the middle atmospheric region.

The author gratefully acknowledges support by the Grant-in-Aid for Scientific research from the Ministry of Education, Science and Culture, Japan, by the Grant-in-aid for 'Computer Experiments and Data Analysis' from the Radio Atmospheric Science Center, Kyoto University, and from the Institute of Space and Astronautical Science. The computation has been carried out at the Data Processing Center of Kyoto University, and at the Integrated Media Environment Experimental Laboratory, Kyoto University. He also appreciates the discussions with Drs. S. Miyahara, F. Vial and J. M. Forbes.

\section{REFERENCES}

Aso, T., T. Nonoyama, and S. Kato, Numerical simulation of semidiurnal atmospheric tides, J. Geophys. Res., 86, 11388-11400, 1981.

Aso, T., S. Iто, and S. KAто, Background wind effect on the diurnal tide in the middle atmosphere, J. Geomag. Geoelectr., 39, 297-305, 1987.

Chapman, S. and R. S. Lindzen, Atmospheric Tides, D. Reidel Pub. Co., Dordrecht, Holland, 1970.

Forbes, J. M., Atmospheric tides 1. Model description and results for the solar diurnal component, J. Geophys. Res., 87(A7), 5222-5240, 1982a.

Forbes, J. M., Atmospheric tides 2. The solar and lunar semidiurnal components, J. Geophys. Res., 87(A7), 5241-5252, 1982b.

Forbes, J. M. and F. Vial, Monthly simulation of the solar semidiurnal tide in the mesosphere and lower thermosphere, J. Atmos. Terr. Phys., 51(7/8), 649-661, 1989.

Haltiner, G. J. and R. T. Williams, Numerical Prediction and Dynamic Meteorology, 477pp., John Wiley \& Sons, New York, 1980.

Holton, J. R., The Dynamic Meteorology of the Stratosphere and Mesosphere, American Meteor. Soc., Boston, Mass., U.S.A., 1975.

LINDZEN, R. S. and S.-S. HONG, Effects of mean winds and horizontal temperature gradients on solar and lunar semidiurnal tides in the atmosphere, J. Atmos. Sci., 31(5), 1421-1446, 1974.

Longuet-Higgins, F. R. S., The eigenfunctions of Laplace's tidal equations over a sphere, Phil. Trans. Roy. Soc. Lonḋon $A, \mathbf{2 6 2}, 511-607,1968$.

Matsuno, T., Numerical integrations of the primitive equations by a simulated backward difference method, $J$. Meteor. Soc. Japan, 44(1), 76-84, 1966.

MrYahara, S., Zonal mean winds induced by solar diurnal tides in the lower thermosphere, J. Meteor. Soc. Japan, 59(3), 303-319, 1981. 
Richmond, A. D., Energy relations of atmospheric tides and their significance to approximate methods of solution for tides with dissipative forces, J. Atmos. Sci., 32, 980-987, 1975.

VIAL, F., Numerical simulation of atmospheric tides for solstice conditions, J. Geophys. Res., 91(A8), 8955-8969, 1986.

Vial, F., J. M. Forbes, and S. MiYahara, Some transient aspects of tidal propagation, J. Geophys. Res., 96(A2), 1215-1224, 1991.

Wu, D.-H., S. Miyahara, and T. Mryoshi, A nonlinear simulation of the thermal diurnal tide, J. Atmos. Terr. Phys., 51(11/12), 1017-1030, 1989. 